\title{
PREVENTIVE MEASURES OF NEURODEGENERATION.
}

M. Usha Rani

\section{Associate Professor. Department of Physiology, Andhra Medical College. Visakhapatnam, Andhra Pradesh.}

\section{CORRESPONDING AUTHOR:}

Dr.M. Usha Rani,

Associate Professor,

Department of Physiology,

Andhra Medical College,

Visakhapatnam, Andhra Pradesh.

E-mail: ushaprasad6@gmail.com

\begin{abstract}
The present article deals with different measures which will prevent the 'NEURODEGENERATION' in the neurons of the central nervous system. The neurodegenerative diseases are characterized by conformational changes in the proteins that result in misfolding, aggregation and intra or extra-neuronal accumulation of amyloid fibrils. To prevent this pathology, we have different 'TOOLS' which are elaborated in the main article below. The goals of Medicine are to promote health, to preserve health, to restore health when it is impaired and to minimize suffering and distress. These goals are embodied in the word "PREVENTION". Successful prevention depends upon a knowledge of causation, dynamics of transmission, identification of risk factors and risk groups, availability of prophylactic or early detection and treatment measures, an organization for applying these measures to appropriate persons or groups and continuous evaluation of and development of procedures applied. Prevention framework includes Primary - true prevention, Secondary - early detection\& screening, Tertiary - doing the best possible in established disease. Effective treatment or intervention for people identified through early detection with evidence of early treatment leading to better outcome than late treatment. Neuroprotection is the mechanisms and strategies used to protect against neuronal injury or degeneration in the Central Nervous System. The goal of neuroprotection is to limit neuronal dysfunction/death after CNS injury and attempt to maintain the highest possible integrity of cellular interactions in the brain resulting in an undisturbed neural function.
\end{abstract}

KEY WORDS: Prevention, Neurodegeneration, Tools.

INTRODUCTION: Neurodegenerative disease has become one of the most exciting and well researched areas of neuroscience in the past few years. This is due to the growing awareness of dementia within our society which is largely caused by diseases such as Alzheimer's. Neurodegeneration itself is basically the progressive loss of neurons via apoptosis, structural abnormalities, or a general failure to function. More than 600 disorders afflict the nervous system. Many neurodegenerative diseases including Parkinson's, Alzheimer's, and Huntington's occur as a result of neurodegenerative processes. As research progresses, many similarities appear which relate these diseases to one another on a sub-cellular level. Discovering these similarities offers hope for therapeutic advances that could ameliorate many diseases simultaneously. There are many parallels between different neurodegenerative disorders including atypical protein assemblies as well as induced cell death. Neurodegeneration can be found in many different levels of neuronal circuitry ranging from molecular to systemic. 


\section{TOOLS:}

\section{- Molecular chaperones :}

Molecular chaperones provide a first line of defense against misfolded, aggregation-prone proteins and are among the most potent suppressors of neuro-degeneration known for animal models of human disease.

Molecular chaperones are neuroprotective because of their ability to modulate the earliest aberrant protein interactions that trigger pathogenic cascades. Eg. CEP-1347 (a semi synthetic derivative of the fermentation product K-252a).

Molecular Chaperones inhibit c-JNK pathway(Jun $\mathrm{N}$ terminal kinase pathway)Inhibition of Neuronal cell death. CEP-1347 has the potential of not only retarding disease progression but also reversing the severity of symptoms by improving the function of surviving neurons. The possible importance of the JNK pathway in neurodegenerative diseases such as Alzheimer's and Parkinson's diseases provides a rationale for the use of CEP-1347 for the treatment of these diseases. Activation of JNK pathway is critical for naturally occurring neuronal cell death during development as well as the pathological neuronal cell death of neurodegenerative diseases. The JNK path way is mitogen-activated-protein kinase (MAPK) pathway that is activated in response to many extracellular stimuli and different forms of environmental stress. The MAPK pathway is organized as a cascade of at least 3 kinases. The JNK pathway can be activated by small G- proteins, such as Cdc42\&Rac members of the RHO family GTPases. In the neurons, the MLK family of kinases serves as the major MAPKKS and phosphorylates MKK4 \& MKK7, which in turn phosphorylate the JNKS. Activation of JNK pathway induces the expression of BH3-only proteins members ofBcl2-2 family which are critical upstream regulators of neuronal apoptosis. (See the flow chart below).

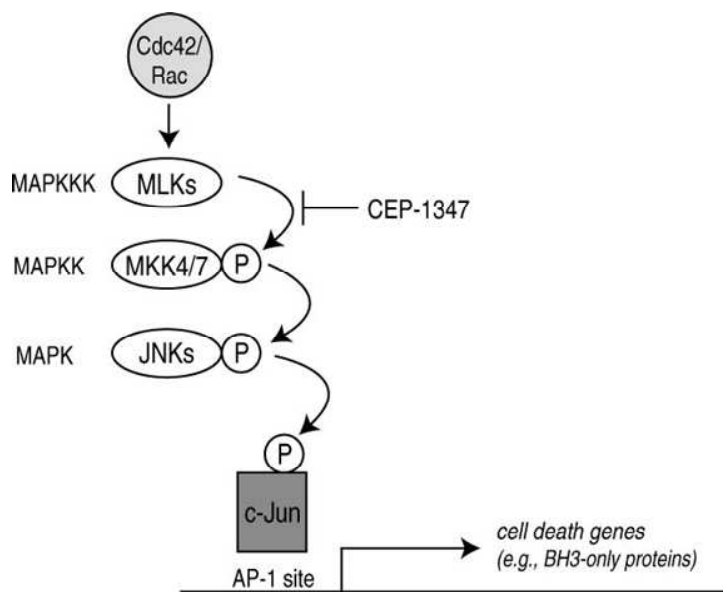

CD200 \& CD200R: CD200 is a human protein encoded by the CD200gene.The protein encoded by this gene is a type 1 membrane glycoprotein, which contains 2 immunoglobulin domains \& thus belongs to the immunoglobulin super family. CD200R is an important inhibitory receptor present on microglia, actively maintains microglia in a quiescent state through its interaction with CD200, a trans-membrane glycoprotein expressed on neurons.(See the diagram below). CD200 \& CD200R dysfunction exacerbates microglial activation and dopaminergic neurodegeneration in a rat model of Parkinson's disease. 
Based on our recent findings that VEGF-B, is a potent protective/survival factor for both the neuronal and vascular systems, which are two critical components in most neurodegenerative disorders. VEGF mediated signaling could contribute to astroglial activation \& inflammatory reactions.

ROLE OF ASTROCYTES IN NEURODEGENERATION: Astrocytes play a critical role in normal function of the mammalian nervous system. Astrocytes regulate $\mathrm{K}+$ buffering, glutamate clearance, brain antioxidant defense, close metabolic coupling with neurons, and modulation of neuronal excitability. In numerous pathological states, such as AD (Alzheimer's Disease), PD (Parkinson's disease), ALS (Amyotrophic lateral Sclerosis) and astrocytes are involved in both exacerbation of damage (reactive astrocytes $2 \%$ present in the old age tissues) and neuroprotective mechanisms. They support neurons in many ways, all of which are essential for repair and regeneration. Disturbances in astrocytic functions are implicated in neurodegenerative diseases pathogenesis, therefore, modulation of astrocyte functioning may prove to be an efficient therapeutic strategy in many chronic CNS disorders.

\section{ROLE OF CD200-CD200R IN NEURODEGENERATIVE CHANGES.}

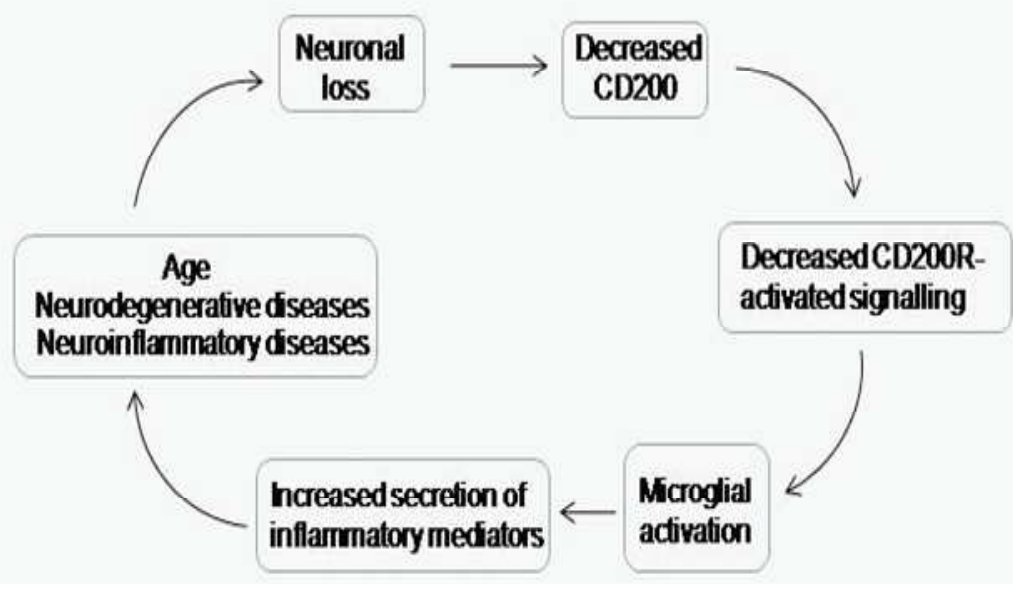

VEGF: (VASCULAR ENDOTHELIAL GROWTH FACTOR)

ROLE OF CONNEXIN HEMI CHANNELS IN NEURODEGENERATION: Dysfunction of astroglial and microglial hemichannels, as well as gap junction channels, are likely mechanisms commonly elicited in all brain diseases associated with inflammatory responses. Therefore, normalization of connexin- and pannexin-based channel dysfunctions should confer tissue protection, improve quality of life, and extend survival of patients suffering acute or chronic brain inflammatory responses. Thus, it is proposed that chronic or acute processes of neurodegeneration might be prevented by blocking glial and neuronal hemichannels. Prevention might also be accomplished by reducing the effects of soluble factors (i.e., glutamate, ATP, prostaglandins, and cytokines) accumulated in the microenvironment of the inflamed CNS. 
ROLE OF ACID SENSING ION CHANNELS [ASICs] IN NEURODEGENERATION: ASICs represent new biological components in peripheral sensory and CNS neurons. Increasing evidence indicates the involvement of these channels in both physiological and pathological processes of CNS (Grunder \& Chen, 2010). Therefore, targeting these channels may provide novel and effective therapeutic interventions for a number of CNS diseases.

\section{ROLE OF DIET IN NEURODEGENERATION}

\section{DHA: DOCOSA HEXAENOIC ACID.}

It is relevant that DHA (Family of $n-3$ polyunsaturated fatty acids) is the most unsaturated fatty acid in our organism and is found specifically concentrated in the brain, retina and sperms of higher animals (Uauy et al., 2001). DHA, when provided by the diet, comes mainly from marine organisms such as fish (fatty or blue species), shellfish, and algae (Horrocks et al,2004).DHA plays a relevant role in the preservation of both the histology and physiology of the neuronal tissue as the individual ages, by preserving the nervous system functions among which memory and learning are the most remarkable (Lukiw \& Bazan, 2008).

\section{CALORIE RESTRICTION:}

Calorie restriction induces sirtuins, silent information regulator proteins that regulate life span, enable DNA repair, protect DNA, and improve the survival of the most abundant antioxidants found in the diet, polyphenols have shown neuroprotective effects in studies over the past decade neurons.

Researchers believe that coenzyme Q10, a fat-soluble compound primarily synthesized in the body and also consumed in the diet, may have the potential to affect the course of neurological disease in which mitochondrial function is impaired and oxidative stress and damage are present

\section{POLYPHENOLS:}

The most abundant antioxidants found in the diet, polyphenols have shown neuroprotective effects in studies over the past decade. It appears that the mechanisms of action for dietary polyphenols extend beyond their antioxidant activity. These include activities such as iron chelation, scavenging radicals, activating survival genes and cell signaling pathways, and regulating mitochondrial function and possibly of the ubiquitin-proteasome system.

4. CURCUMIN: Curcumin appears to slow the formation of-and possibly even destroyaccumulated plaque deposits at the root of AD. Curcumin significantly lowered oxidized proteins and interleukin-1beta, a proinflammatory cytokine, in Alzheimer transgenic mice brains, according to a 2001 study in The Journal of Neuroscience, Greg Cole, PhD, a professor of medicine and neurology at UCLA, who has studied the effects of curcumin on AD, reports that curcumin is a potent antiamyloid drug with an established safety profile that has reversed cognitive defects in animal models. 


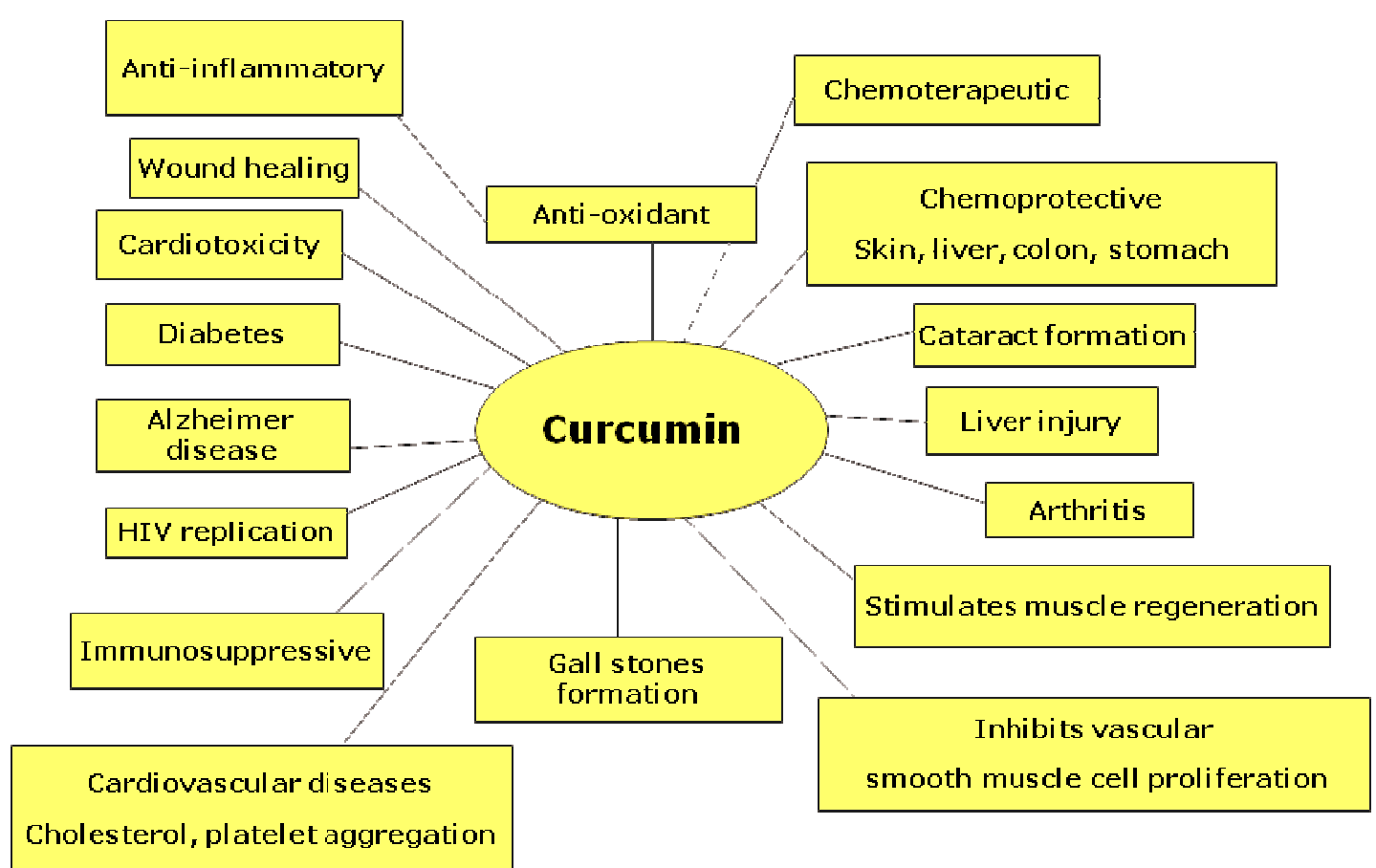

5. MEDITERRANEAN DIET:[Balanced diet of low fat, moderate carbohydrate, and good protein.]

Along with a host of disease-prevention benefits, the Mediterranean diet has also been linked with a lower risk of AD. Researchers discovered that higher adherence to the Mediterranean diet was associated with a lower risk of AD, possibly due to the reduction in inflammation and an antioxidant effect.

6. DIETARY DAMAGE: while nutritional strategies that reduce inflammation and oxidative stress appear to hold promise for preventing neurodegenerative disease, it's important to note that some nutritional factors may actually enhance brain inflammation. Obesity seems to be on the top of the list of concerns. Studies have shown that people with higher levels of adiposity are at higher risk for both future PD and $\mathrm{AD}$ and that central adiposity is related to cognitive decline and dementia.

"Body fat promotes inflammation. Body fat may store toxins. A fatter person even has a smaller hippocampus," says Perlmutter of links between obesity and neurodegeneration.

\section{ACHILLEA FRAGRINTISSIMA [AF]:(LAVENDER COTTON-A DESERT PLANT)}

Extract of Achillea fragrantissima Down regulates ROS Production and Protects Astrocytes from Oxidative-Stress-Induced Cell Death. Af extract inhibits H2O2- and $\mathrm{ZnCl} 2$-induced ROS (Reactive oxygen species) generation. AF extract reduces 2,2'-azobis(amidinopropane) (ABAP)-mediated peroxyl radicals levels in astrocytes (ROS-reactive 02 species.). 


\section{REVIEW ARTICLE}

\section{ACHILLEA FRAGRINTISSIMA:}

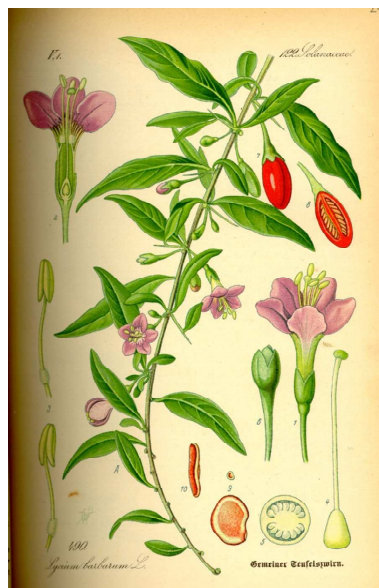

\section{WOLF BERRY FRUIT (raw \& dry):}

\section{WOLFBERRY}

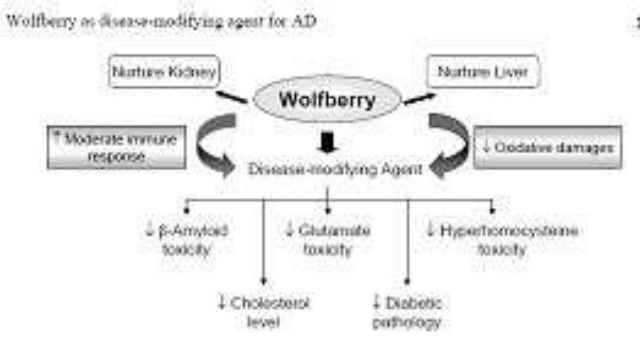

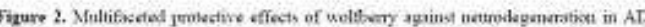

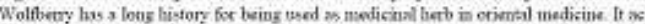

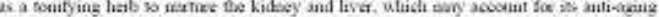

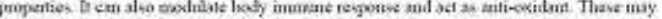
be respotsible for in netrogeonstive eftests agting sevenal condinass teissod 10 AD pathogetiesis. Wollterry is able to attemate Af pepcidt rosicity and sfuramote

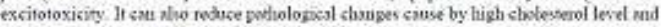
disbetes mellitus. All of these beteficisl effests ensble wollbery to become, posertal fivare-modifying azent for the pren vatim or treatment of $A D$ 

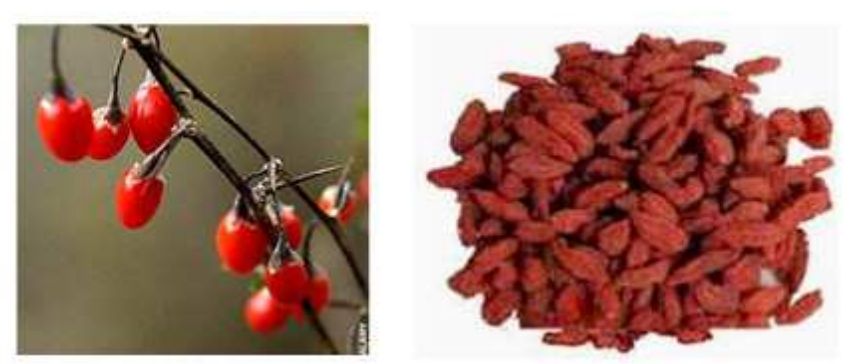

\section{REFERENCES:}

1. Leo H.Wang, Cagri G. Besirli, and Eugene M. Johnson, Jr: MIXED-LINEAGE KINASES: A Target for the Prevention of Neurodegeneration.

2. By Sharon Palmer, RD: Smart Eating- How Diet May Help Preserve the Brain Vol. 11 No. 7 P. 24 July 2009 Issue

3. Chu Xiang-Ping1, Wang John Q.1 and Xiong ZhGang2: Acid Sensing Ion Channels in Neurodegenerative Diseases: Potential Therapeutic Target.

4. Alla B. Salmina et al: .Alteration of Neuron-Glia Interactions in Neurodegeneration: Molecular Biomarkers and Therapeutic Strategy.

5. Anne-Marie Miller1, Brian F. Deighan1, Eric Downer1, Anthony Lyons1Petra HenrichNoack1, Yvonne Nolan2 and Marina A. Lynch1: .Analysis of the Impact of CD200on Neurodegenerative Diseases.

6. Emmanuel Sturchler, Daniel Feurstein, Patricia McDonald and Derek Duckett: ASK1 and Its Role in Neurodegenerative Diseases.

7. Xuri Li, Anil Kumar, Chunsik Lee, Zhongshu Tang, Yang Li, Pachiappan Arjunan, Xu Hou and Fan Zhang: .Can VEGF-B Be Used to Treat Neurodegenerative Diseases?

8. Rodrigo Valenzuela B.1 and Alfonso Valenzuela B.2: Docosahexaenoic Acid (DHA), in the Prevention and Treatment of Neurodegenerative Diseases.

9. Nadia Sadli1, Nayyar Ahmed1, M. Leigh Ackland2, Andrew Sinclair3, Colin J. Barrow4 and Cenk Suphioglu1: Effect of Zinc and DHA on Expression Levels and PostTranslational Modifications of Histones $\mathrm{H} 3$ and H4 in Human Neuronal Cells.

10. Anat Elmann1, Alona Telerman1, Sharon Mordechay1,Hilla Erlank1, Miriam Rindner1, Rivka Ofir2 and Elie Beit-Yannai3: .Extract of Achillea fragrantissima Down regulates ROS production and protects Astrocytes from Oxidative-Stress-Induced Cell Death.

11. Chirakkal V. Krishnan1,2, Merrill Garnett1 and Frank Antonawich11Garnett McKeen Laboratory, Inc, Bohemia: Free Radicals in Neurodegenerative Diseases: Modulation by Palladium $\alpha$-Lipoic Acid Complex.

12. Diana Gallego, Manuel Rojas and Camilo Orozco: .Free Radicals, Neuronal Death and Neuroprotection.

13. Luca Lovrečić, Aleš Maver and Borut Peterlin:. Genome Profiling and Potential Biomarkers in Neurodegenerative Disorders.

14. Alexander Shpakov, Oksana Chistyakova,Kira Derkach and Vera Bondareva: Hormonal Signaling Systems of the Brain in Diabetes Mellitus

15. Humberto Mestre and Antonio Ibarra: .Immunization with Neural-Derived Peptides as a Potential Therapy in Neurodegenerative Diseases. 
16. Jose M. Vidal-Taboada, Nicole Mahy and Manuel J. Rodríguez: Microglia, Calcification and Neurodegenerative Diseases.

17. Paul Bustamante1,2,Gonzalo Solas1 and Karol Grandez1:Neurodegenerative Disease Monitoring Using a Portable Wireless Sensor Device

18. Julie Allyson and Guy Massicotte: Neuropathological Disorders and Calcium Independent Forms of Phospholipase A2 Activities in the Brain.

19. Selva Rivas-Arancibia, Cesar Gallegos-Ríos,Nancy Gomez-Crisostomo, Ever FerreiraGarcidueñas, Dulce Flores Briseño, Luz Navarro and Erika Rodríguez-Martínez: Oxidative Stress and Neurodegenerative Disease.

20. Céline Domange1, Alain Paris2, Henri Schroeder3 and Nathalie Priymenko1,4: Power of a Metabonomic Approach to Investigate an Unknown Nervous Disease.

21. Niyazi Acer1, Ahmet Tuncay Turgut2,Yelda Özsunar3 and Mehmet Turgut4: Quantification of Volumetric Changes of Brain in Neurodegenerative Diseases Using Magnetic Resonance Imaging and Stereology.

22. George E. Barreto1, Janneth Gonzalez1,Francisco Capani2 and Ludis Morales1:Role of Astrocytes in Neurodegenerative Diseases.

23. Juan A. Orellana1,2, Christian Giaume3 and Juan C. Sáez1,4: Role of ConnexinHemichannels in Neurodegeneration.

24. Kentaro Yomogida, Shumi Yoshida-Yamamoto and Hiroshi Doi: .Roles of Microtubules in Maintenance of Nerve Cell Networks.

25. Garth F. Hall: What is the Link Between Protein Aggregation and Interneuronal LesionPropagation in Neurodegenerative Disease?

26. David Vauzour, Ana Rodriguez-Mateos, Giulia Corona, Maria Jose Oruna-Concha and Jeremy P. E. Spencer : Polyphenols and Human Health: Prevention of Disease and Mechanisms of Action .

27. Carol Brayne: Screening for neurodegenerative disorders.

28. Charles Ramassamy and Stéphane Bastianetto: Recent Advances on Nutrition and the Prevention of Alzheimer's Disease, 2010: 169-185 ISBN: 978-81-7895-470-7.

29. Raymond Chuen-Chung Chang1,2,3, Yuen-Shan Ho1, Man-Shan Yu1 and Kwok-Fai So1,2,3: Medicinal and nutraceutical uses of wolfberry in preventing neurodegeneration in Alzheimer's disease.

30. Cork neuroscience group $>$ research themes $>$ neurodegenration and ageing.

31. Peter M.Douglas and Andrew Dillin :Protein homeostasis and aging in neurodegeneration.

32. Xiao-Guang Luo, Jian-Qing Ding and Sheng-Di Chen . Microglia in the aging brain: relevance to neurodegeneration.

33. James Joseph, Greg Cole, Elizabeth Head, and Donald Ingram: Nutrition, Brain Aging, and Neurodegeneration, Journal of neuroscience,October,2009

34. John V.Hindl: Ageing, neurodegeneration and Parkinson's disease OXFORD JOURNALS.

35. NATURE: International weekly journal of science Nature.com

36. Diseases Hindawi Publishing corporation : neurodegenerative and neurobehavioral diseases.

37. Chittaranjan Andrade and Rajiv Radhakrishnan: The prevention and treatment of cognitive decline and dementia: An overview of recent research on experimental treatments. Indian J Psychiatry. 2009 Jan-Mar; 51(1): 12-25. 
38. 38. The Source for Objective Science-based DHA/EPA Omega-3 Information: DHA for Optimal Brain and Visual Functioning .

39. Wikipedia, the free encyclopedia : File:Connexon and connexin structure.

40. Jenna Gregory: Cambridge Medicine journal [CMJ] Our lives in the balance: Cancer or Neurodegeneration.

41. Brian P. Head, et al:Loss of Caveolin-1 Accelerates neurodegeneration and Aging.

42. From Wikipedia, the free encyclopedia: Neurodegeneration

43. MNT-MEDICAL NEWS TODAY: What Is Neuroprotection?

44. Nicolas G. Bazan1:Neuroprotectin D1-mediated anti-inflammatory and survival signaling in stroke, retinal degenerations, and Alzheimer?s disease.

45. Natural News.com, Real News powered by the people,Naturally

46. Rastogi M, Ojha RP, Prabu PC, Devi BP, Agrawal A, Dubey GP.: Prevention of age associated neurodegeneration and promotion of healthy brain ageing in female Wistar rats by long term use of bacosides. Biogerontology. 2011 Dec 6 .

47. Stacey et al ; The Promise of Neuroprotective Agents in Parkinson's Disease;Front Neurol. 2011; 2: 68. Published online 2011 November 21.

48. HealthyVillage.com-wolf Berry. 